\title{
Two-port pars plana vitrectomy surgery: a prospective interventional case series
}

\begin{abstract}
Background and objective Current literature review fails to disclose any series describing the use of two-port vitrectomy in adult patients. This study was performed to determine the feasibility and efficacy of pars plana vitrectomy surgery using two (rather than three or four)port access for treatment of diabetic patients with nonclearing vitreous haemorrhage due to retinal neovascularization.

Design Interventional prospective case series: to measure ability to allow for longterm resolution of chronic uncomplicated vitreous haemorrhage in diabetic patients, and to study the frequency and nature of complications associated with this technique. Methods Two-port pars plana vitrectomy (with endolaser treatment and membrane delamination if necessary) was performed in a prospective series of 12 consecutive diabetic patients with nonclearing vitreous haemorrhage due to retinal neovascularization.

Results Successful removal of vitreous haemorrhage resulted in all patients. No visually significant intraoperative complications occurred. Best postoperative visual acuity correlated with lenticular and macular perfusion status.

Conclusion Two-port pars plana vitrectomy is an efficient (and potentially safer and faster) alternative to the standard three-port vitrectomy in selected patients.

Eye (2005) 19, 869-872. doi:10.1038/sj.eye.6701671; published online 3 September 2004
\end{abstract}

Keywords: pars plana vitrectomy; retina surgery; two-port pars plana vitrectomy; vitreous haemorrhage; vitreous surgery

\section{Introduction}

Pars plana vitrectomy surgery was introduced in 1968, and initially was performed with one-
M Colucciello

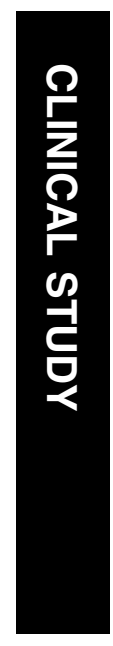

port intraocular access. ${ }^{1}$ The port was made in the superotemporal quadrant, $5 \mathrm{~mm}$ posterior to the limbus (where the pars plana is the widest). A $3.5 \mathrm{~mm}$ incision was made in this region to allow for intraocular access for the Vitrectomy Infusion Suction and Cutting (VISC) instrument, which has a tip diameter of 2.2$2.5 \mathrm{~mm}$. This instrument combined infusion capabilities to maintain intraocular pressure intraoperatively with vitrectomy and suction functions.

One-port access limited the surgeon to unimanual manoeuvres, and there was no possibility for other instrumentation such as scissors, picks, or endolaser. Also, the incision needed to be relatively large, which increased potential for complications. Therefore, divided system access was introduced, ${ }^{2}$ and is now the standard method. Typically, three 20-gauge pars plana ports are used - one for the infusion cannula (usually placed inferotemporally, sometimes superiorly), and two other ports to allow for intraocular access for a variety of intraocular microinstruments (such as fibre optic endoilluminator, microscissors, endolaser, vitrectomy suction and cutting instrument, active and passive extrusion cannulas, and an assortment of microforceps). A four-port system was recently introduced to allow for bimanual intraocular manipulation - the fourth port in this system is for 'chandelier' endoillumination, freeing the surgeon's hand from having to hold an endoilluminator.

Prototypes of 20-gauge combined illumination-irrigation probes were developed in the late 1970s and early 1980s. ${ }^{3,4}$ These authors proposed that the utilization of these probes allowed for the possibility of pars plana vitrectomy with two (rather than three or four) pars plana incisions.

A current literature review failed to disclose any series of adult patients that underwent twoport pars plana vitrectomy. A two-port system for pars plicata vitrectomy lens-sparing
South Jersey Eye Physicians Moorestown

NJ, USA

Correspondence:

M Colucciello

South Jersey Eye Physicians 509 South Lenola Road, Suite \#11 Moorestown, NJ Tel: + 18562340222

Fax: + 18567279518

E-mail: michael@macula.us; retina7@pol.net

Received: 21 March 2004 Accepted: 8 June 2004 Published online:

3 September 2004

Presented at the Scheie Eye Institute/University of Pennsylvania Department of Ophthalmology 129th Anniversary Meeting, May 30, 2003

For further information, visit the website http://www.macula.us 
vitreoretinal surgery in infants has been described. ${ }^{5}$ This current report describes a series of adult diabetic patients with nonclearing vitreous haemorrhage in which a twoport pars plana vitrectomy was performed using a (Storz DP9602) 20-gauge combined endoillumination-irrigation cannula with other intravitreal microinstruments. This series was undertaken to determine the efficacy of twoport vitrectomy in allowing for clearance and long-term resolution of chronic vitreous haemorrhage.

\section{Materials and methods}

A total of 12 consecutive patients were selected to undergo two-port pars plana vitrectomy between March and August 2002 who met the criteria of chronic (greater than 3 months) nonclearing uncomplicated (ie no associated tractional retinal detachment) vitreous haemorrhage.

The surgery was performed as follows: after initiation of general anaesthesia with either endotracheal intubation or laryngeal mask airway, a conjunctival peritomy was performed $1 \mathrm{~mm}$ posterior to the limbus superotemporally and superonasally. Horizontal conjunctival relaxing incisions were made, and haemostasis was obtained. Sclerotomy sites of $1.4 \mathrm{~mm}$ thickness were made $3.5 \mathrm{~mm}$ posterior to the limbus superotemporally and superonasally in pseudophakic eyes ( $4.0 \mathrm{~mm}$ posterior to the limbus in phakic eyes) to accommodate 20-gauge $(0.9 \mathrm{~mm})$ diameter instruments. The sclerotomy incisions were then 'guarded' with a single 7-0 vicryl suture, which was not secured, but was 'looped' three times and was set aside (the portion of the suture spanning the sclerotomy incision was pulled from the incision and also set aside). This is done to allow for quick closure of the sclerotomy sites at the end of the case to prevent hypotony.

The sclerotomy sites allowed for intraocular access for a variety of 20-gauge diameter microinstruments. A combination endoilluminator/irrigation cannula was always inserted in one of the ports. The infusion solution was attached to the irrigation cannula portion of the hand piece with tubing, which had male adapters on each end. The intraocular infusion was begun when the tip of the endoilluminator/irrigation probe was viewed in the vitreous cavity, and infusion pressures were generally set between 50 and $60 \mathrm{mmHg}$ due to the resistance in the system. The central retinal artery always showed $100 \%$ patency (ie intraocular pressures were not high at these infusion pressures). The other sclerotomy site was utilized for intraocular access for the vitrectomy suction/cutting device, silicone-tipped active aspiration cannula, retinal pick, microscissors, or curved endolaser probe. Using the binocular indirect ophthalmomicroscope (BIOM) lens system for wide-field visualization, a 'core' vitrectomy was performed. The mid-peripheral vitreous cortex was then incised inferiorly, and this incision was carried out for 360 (switching hands during the manoevre if necessary). If choroidal folds were noted (signifying low intraocular pressure), then either the intraocular infusion pressure was raised or aspiration pressures were reduced. Flow was also sufficient to stop neovascular bleeding by increasing the intraocular infusion pressure as one does with a separate infusion line. The anterior vitreous cortex was then 'shaved' for $360^{\circ}$; the performance of this manoevre was enhanced due to the wide-field viewing system. The posterior vitreous cortex was then cut and aspirated with the vitrectomy suction and cutting instrument; unimanual delamination and segmentation of the posterior vitreous cortex (if necessary) was performed with the retinal pick and micro scissors. Preretinal haemorrhage was aspirated with the siliconetipped active extrusion cannula. Scatter endolaser panretinal photocoagulation was performed with the curved endolaser probe (which can be accomplished without switching hands and removing the endoilluminator/irrigating cannula since the instrument is curved). Examination of the fundus periphery to detect iatrogenic retinal tears was then accomplished with scleral depression and viewing through the BIOM lens; retinal tears were sealed with endolaser

photocoagulation. The instruments were withdrawn and the sclerotomy sites were permanently closed with the vicryl sutures that had been preplaced. Anaesthetic, antibiotic, and anti-inflammatory agents were delivered in a subtenons retrobulbar manner, and the conjunctival peritomy sites were closed with $6-0$ plain gut suture material. Topical cycloplegic, antibiotic, and antiinflammatory agents were given.

\section{Results}

The characteristics and outcomes of the patients are summarized in Table 1 ( $\mathrm{VHb}$ indicates vitreous haemorrhage, PDR indicates proliferative diabetic retinopathy, CRVO indicates central retinal vein occlusion). The posterior vitreous cortex was attached preoperatively in all patients except in patient number 8 , who was being operated on for a rebleed and haemolytic glaucoma. Scissors delamination and segmentation of the posterior vitreous cortex was necessary in patient 2 .

Best postoperative visual acuity was dependent on lenticular and macular perfusion status (assessed with intravenous fluorescein angiography). All patients had complete clearance of vitreous haemorrhage without evidence for rebleed as of February 2004 (a follow-up of 17-23 months). 
Table 1 Characteristics and outcomes of patients in the study

\begin{tabular}{|c|c|c|c|c|c|c|}
\hline Patient & Age (years) & Lens status & VHB cause & Pre-op VA & Post-op VA & Associated pathology \\
\hline 1 & 79 & PC IOL & PDR & $20 / 200$ & $20 / 50$ & Macular ischaemia \\
\hline 2 & 49 & NS & PDR & $1 / 400$ & $20 / 60$ & Macular ischaemia \\
\hline 3 & 44 & NS & PDR & $1 / 1200$ & $20 / 80$ & Macular ischaemia \\
\hline 4 & 64 & NS & PDR & $2 / 200$ & $20 / 30$ & (2) Peripheral retinal tears (intraop) \\
\hline 5 & 78 & PC IOL & PDR & $1 / 200$ & $20 / 25$ & \\
\hline 6 & 63 & PC IOL & PDR & HM 2' & $20 / 400$ & Macular ischaemia, Quadrantanopia s/p CVA \\
\hline 7 & 51 & NS & PDR & $3 / 200$ & $20 / 30$ & Nuclear Sclerosis \\
\hline 8 & 55 & NS & PDR & LP & $4 / 200$ & Macular ischaemia, Haemolytic GL \\
\hline 9 & 78 & PC IOL & PDR/CRVO & $1 / 1200$ & $3 / 200$ & Macular ischaemia, GL \\
\hline 10 & 73 & PC IOL & PDR & $20 / 100$ & $20 / 80$ & Macular ischaemia \\
\hline 11 & 82 & PC IOL & PDR & $1 / 400$ & $20 / 70$ & Macular ischaemia \\
\hline 12 & 54 & NS & PDR & HM $2^{\prime}$ & $20 / 25$ & \\
\hline
\end{tabular}

\section{Discussion}

This report describes a prospective interventional case series of 12 patients with uncomplicated nonclearing vitreous haemorrhage. While these cases were described as being accomplished under general anaesthesia, the two-port system exhibits compatibility with local anaesthesia in selected patients. Operative time is relatively short, fewer incisions are made, and the cases are relatively straightforward and shorter in duration (ie two-port vitrectomy in more lengthy, complicated cases requiring extensive delamination or bimanual manipulation may be relatively contraindicated at this point). In addition, there appears to be less potential for complications associated with the creation of the additional sclerotomy incision site, such as retinal tear formation, retinal detachment, retinal incarceration, endophthalmitis, vitreous skirt incarceration, and retinal neovascularization associated with the sclerotomy site (peripheral hyaloid neovascularization).

The recently described 25-gauge three-port vitrectomy system continues to require the creation of three instead of two ports. Intraocular pressure was reported to be $<10 \mathrm{mmHg}$ in eight out of 29 cases where it was measured on the first postoperative day in the 25-gauge no-stitch three-port vitrectomy cases. ${ }^{6}$ Since the ports are sutured closed in the two-port system, no concern of postoperative hypotony from postoperative loss of intravitreal contents exists. Aspiration with the 25-gauge system is less than the 20-gauge system at comparable aspiration pressures due to the decreased diameter of the instruments, therefore, lengthening surgical time. Also, no unique instrumentation, other than the infusing endoilluminator, is necessary to carry out the two-port cases. With the 25-gauge system, separate smaller instrumentation sets need to be purchased.

Since the ports in a two-port system are located in a superior location (ie there is no inferior port as exists in a three-port system), a retinal detachment due to a tear located near the ports can likely be treated with a pneumatic retinopexy in most cases. The disadvantage of this system is that patient cases requiring extensive delamination and/or bimanual manipulation (such as en bloc dissection of the posterior vitreous cortex or delamination of extensive proliferative vitreoretinopathy tissue) may not be optimal candidates. Scissors delamination and segmentation of the posterior vitreous cortex was accomplished without complication in one of the 12 described cases. Delamination of the internal limiting membrane in macular hole cases may be accomplished with one hand, so this surgical procedure appears to be compatible with the two-port technique. In any case, if a given surgery does not appear to be amenable to the two-port technique intraoperatively, it can easily be converted to a three-port technique. The intraocular pressure can be maintained during the production of the third port by plugging the two existing ports. Conversion to three ports was not necessary in any of the cases described.

This report has described the first series of patients in which two-port pars plana vitrectomy surgery was performed. It appears to be an effective, safe, and efficient alternative to the three-port system. Since there are fewer ports to be created, in selected patients, this procedure is likely to shorten surgical time and has the theoretical advantage of fewer complications compared to systems which use more or larger ports.

\section{References}

1 Machemer R, Parel JM, Buettner H. A new concept for vitreous surgery. 1. Instrumentation. Am J Ophthalmol 1972; 73: 1.

2 Machemer R. A new concept for vitreous surgery. 7. Two instrument techniques in pars plana vitrectomy. Arch Ophthalmol 1974; 92: 407. 
3 Poole TA, Sudarsky RD. A 20-gauge illumination and irrigation instrument for pars plana vitrectomy. Am J Ophthalmol 1979; 88: 1093.

4 Meyers SM, Bonner RF, Leighton SB. Combined

illumination-irrigation 20 gauge probes for vitrectomy. Arch Ophthalmol 1982; 100: 622.
5 Maguire AM, Trese MT. Lens-sparing vitreoretinal surgery in infants. Arch Ophthalmol 1992; 110: 284

6 Fujii GY, de Juan E, Humayun M, Pieramici DJ, Chang TS, $\mathrm{Ng}$ E et al. Initial experience using the transconjunctival sutureless vitrectomy system for vitreoretinal surgery. Ophthalmology 2002; 109: 1814 